\title{
Influence of Random Variation in Volume Fraction of Fiber on Stochastic Property of Equivalent Elastic Property of Unidirectional Fiber Reinforced Composite Material ${ }^{*}$
}

\author{
Sei-ichiro SAKATA ${ }^{* *}$ and Fumihiro ASHIDA ${ }^{* *}$ \\ **Shimane University, \\ 1060 Nishikawatsu-cho, Matsue, Shimane, Japan \\ E-mail: sakata@ecs.shimane-u.ac.jp
}

\begin{abstract}
This paper describes stochastic analysis on a homogenized elastic property of a unidirectional fiber reinforced composite material considering uncertainty in material and geometrical properties of component materials. The influence of the microscopic uncertainties on the stochastic characteristics of a homogenized elastic property is analyzed with the perturbation-based analysis using the equivalent inclusion method. Especially, influence of a volume fraction variation on the stochastic characteristics is discussed in this paper. Also, as an example of a design considering the influence of a volume fraction variation, a limit of the random variation of the volume fraction of fiber for controlling a random variation of a homogenized elastic property of a composite to be less than random variations of elastic properties of component materials is discussed.
\end{abstract}

Key words: Stochastic Homogenization, First Order Perturbation, Composite Material, Equivalent Inclusion Method

\section{Introduction}

A stochastic homogenization problem has been analyzed in order to investigate an influence of a microscopic uncertainty in a component material on a homogenized material property in recent. Kaminski ${ }^{(1)}$, Ostojya. ${ }^{(2)}, \mathrm{Xu}^{(3)}$ or Sakata ${ }^{(4)}$ reported several results with respect to the stochastic homogenization problem of composite materials or a heterogeneous material.

In addition to a conventional method using the Monte-Carlo simulation ${ }^{(4)(5)}$, the perturbation-based homogenization method ${ }^{(6)-(9)}$ or an approximation-based stochastic analysis method ${ }^{(10)(11)}$ for solving the stochastic homogenization problem has been reported. Such an approximation-based method will be effective for a stochastic homogenization analysis considering some kinds of random variables, for instance geometry of fiber or inclusions, because an analytical perturbation term cannot be computed for such a random variable in case of using the finite element based homogenization method. On the other hand, the perturbation based approach will be still effective for a large numbers of random variables, since an approximation model will be difficult to be constructed for many random variables. From this viewpoint, Sakata et al. proposed the perturbation-based stochastic

*Received 1 Oct., 2009 (No. 09-0553) [DOI: 10.1299/jmmp.4.435]

Copyright $@ 2010$ by JSME 
homogenization analysis approach using the equivalent inclusion method especially for a geometrical random variation ${ }^{(12)(13)}$.

In the previous reports, only accuracy of the perturbation-based approach has been discussed for a certain volume fraction of inclusions. However, the stochastic characteristics of the homogenized elastic properties will depend on the volume fraction of inclusions and its random variation. This point has not been discussed yet.

In this study, therefore, influence of the volume fraction of fiber and its random variation on the stochastic characteristics of the homogenized elastic property of a unidirectional fiber reinforced composite material is investigated.

At first, outline of the first order perturbation-based stochastic homogenization method using the equivalent inclusion method is introduced. Next, accuracy of the perturbation-based analysis for each volume fraction of fiber is evaluated with a comparison between its result and the result of the Monte-Carlo simulation. Finally, the influence of the volume fraction of fiber and its random variation on the stochastic characteristics of the homogenized elastic properties are discussed with the numerical results.

\section{Perturbation-based Stochastic Homogenization Analysis using the Equivalent Inclusion Method}

A homogenized elastic tensor of composites can be evaluated with using the Mori-Tanaka theory $^{(14)}$ as

$$
\begin{aligned}
\boldsymbol{E}^{E I} & =\boldsymbol{E}_{m}\left\{\boldsymbol{E}_{m}-\left(1-V_{f}\right)\left(\boldsymbol{E}_{m}-\boldsymbol{E}_{f}\right) \boldsymbol{S}\right\}^{-1} \times\left[\boldsymbol{E}_{m}-\left(\boldsymbol{E}_{m}-\boldsymbol{E}_{f}\right)\left\{\boldsymbol{S}-V_{f}(\boldsymbol{S}-I)\right\}\right] \\
& =\boldsymbol{E}_{m} \boldsymbol{X}^{-1} \boldsymbol{Y}
\end{aligned}
$$

where $\boldsymbol{E}^{E I}$ is a homogenized elastic tensor, $\boldsymbol{E}_{f}$ is an elastic tensor of an inclusion, $\boldsymbol{E}_{m}$ is an elastic tensor of matrix, $V_{f}$ is a volume fraction of inclusions, $\boldsymbol{S}$ is the Eshelby tensor. Figure 1 shows a schematic view of an inclusion and a unidirectional fiber reinforced composite plate. As shown in Fig.1 (a), $a_{3} \gg a_{1}, a_{2}$ is assumed.
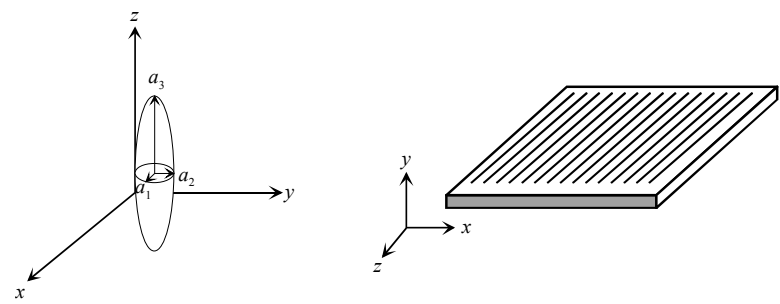

$\begin{array}{lll}\text { (a) dimension of inclusion } & \text { (b) schematic view of unidirectional composite plate }\end{array}$

Fig.1 Dimension of an inclusion

An observed value of the homogenized elastic tensor considering a small random variation $\alpha$ can be approximated with using the first order perturbation method as

$$
\boldsymbol{E}^{E I *} \approx \boldsymbol{E}^{E I 0}+\boldsymbol{E}^{E I 1} \alpha
$$

where $\boldsymbol{E}^{E I i}$ shows the $i$ th order perturbation term. The first order perturbation term of the homogenized elastic tensor considering a random variation of Young's modulus of fiber $E_{f}$, Poisson's ratio of matrix $v_{m}$ or the volume fraction of inclusions $V_{f}$ can be expressed as follows ${ }^{(12)(13)}$.

$$
\begin{gathered}
\left.\boldsymbol{E}^{E I 1}\right|_{E_{f}}=\boldsymbol{E}_{m}\left[-\boldsymbol{X}^{-1}\left(1-V_{f}\right) \boldsymbol{E}_{f} \boldsymbol{S} \boldsymbol{X}^{-1} \boldsymbol{Y}+\boldsymbol{X}^{-1} \boldsymbol{E}_{f}\left\{\boldsymbol{S}-V_{f}(\boldsymbol{S}-\boldsymbol{I})\right\}\right] \\
\left.\boldsymbol{E}^{E I 1}\right|_{V_{m}}=\overline{\boldsymbol{E}_{m}} \boldsymbol{X}^{-1} \boldsymbol{Y}-\boldsymbol{E}_{m} \boldsymbol{X}^{-1}\left\{\overline{\boldsymbol{E}_{m}}-\left(1-V_{f}\right) \overline{\boldsymbol{E}_{m}} \boldsymbol{S}-\left(1-V_{f}\right)\left(\boldsymbol{E}_{m}-\boldsymbol{E}_{f}\right) \overline{\boldsymbol{S}}\right\} \boldsymbol{X}^{-1} \boldsymbol{Y} \\
+\boldsymbol{E}_{m} \boldsymbol{X}^{-1}\left[\overline{\boldsymbol{E}_{m}}-\overline{\boldsymbol{E}_{m}}\left\{\boldsymbol{S}-V_{f}(\boldsymbol{S}-\boldsymbol{I})\right\}-\left(\boldsymbol{E}_{m}-\boldsymbol{E}_{f}\right)\left(\overline{\boldsymbol{S}}-V_{f} \overline{\boldsymbol{S}}\right)\right] \\
\left.\boldsymbol{E}^{H 1}\right|_{V_{f}}=\boldsymbol{E}_{m}\left[-\boldsymbol{X}^{-1} V_{f}\left(\boldsymbol{E}_{m}-\boldsymbol{E}_{f}\right) \boldsymbol{S} \boldsymbol{X}^{-1} \boldsymbol{Y}+\boldsymbol{X}^{-1}\left(\boldsymbol{E}_{m}-\boldsymbol{E}_{f}\right) V_{f}(\boldsymbol{S}-\boldsymbol{I})\right]
\end{gathered}
$$

where $\overline{\boldsymbol{E}_{m}}$ is the perturbation term related to the elastic tensor of matrix, which is 
expressed as the following form.

$$
\begin{aligned}
& \overline{\boldsymbol{E}_{m}}=\left[\begin{array}{cccccc}
\eta_{m} & \beta_{m} & \beta_{m} & 0 & 0 & 0 \\
& \eta_{m} & \beta_{m} & 0 & 0 & 0 \\
& & \eta_{m} & 0 & 0 & 0 \\
& & & \gamma_{m} & 0 & 0 \\
& \text { Sym. } & & & \gamma_{m} & 0 \\
& & & & & \gamma_{m}
\end{array}\right] \\
& \eta_{m}=\frac{-2 v_{m}{ }^{3}+4 v_{m}{ }^{2}}{\left(v_{m}+2 v_{m}{ }^{2}-1\right)^{2}} E_{m}, \beta_{m}=\frac{E_{m} v_{m}\left(1+2 v_{m}{ }^{2}\right)}{\left(1-v_{m}-2 v_{m}{ }^{2}\right)^{2}} \\
& \gamma_{m}=\frac{-E_{m} v_{m}}{2\left(1+v_{m}+2 v_{m}^{2}\right)}
\end{aligned}
$$

In this paper, it is assumed that inclusions and matrix are isotropic materials. $\bar{S}$ is the perturbation term related to the Eshelby tensor.

In case of an orthotropic material, an equivalent elastic constants can be also computed as $^{(15)}$

$$
\begin{aligned}
& E_{x}{ }^{E I}=\frac{1}{C_{11}{ }^{E I}}, \quad E_{y}{ }^{E I}=\frac{1}{C_{22}{ }^{E I}}, \quad E_{z}^{E I}=\frac{1}{C_{33}{ }^{E I}} \\
& v_{y z}{ }^{E I}=-E_{y}{ }^{E I} C_{23}{ }^{E I}, \quad v_{z x}{ }^{E I}=-E_{z}{ }^{E I} C_{31}{ }^{E I}, \quad v_{x y}{ }^{E I}=-E_{x}{ }^{E I} C_{12}{ }^{E I} \\
& G_{y z}^{E I}=\frac{1}{C_{44}{ }^{E I}}, \quad G_{z x}^{E I}=\frac{1}{C_{55}{ }^{E I}}, \quad G_{x y}{ }^{E I}=\frac{1}{C_{66}{ }^{E I}}
\end{aligned}
$$

where $\boldsymbol{C}^{H}$ is a homogenized compliance matrix, which can be computed as the inverse of the homogenized elastic tensor $\boldsymbol{E}^{H}$.

Using the result of the perturbation analysis, the observed homogenized compliance can be approximated as

$$
C_{i j}^{E I^{*}} \approx\left\{\begin{array}{c}
\frac{Q^{0}{ }_{i j}+Q^{1}{ }_{i j} \alpha+\cdots}{R^{0}+R^{1} \alpha+\cdots}: i, j=1 \sim 3 \\
\frac{1}{E_{i j}{ }^{E I^{*}}} \quad: i, j=(4,4),(5,5),(6,6)
\end{array}\right.
$$

where $Q^{k}{ }_{i j}$ and $R^{k}$ are coefficients computed from the perturbation terms of the homogenized elastic tensor, and $\alpha$ is a random variable. The approximated form of the observed homogenized compliance with the perturbation method is expressed as

$$
C_{i j}{ }^{E I^{*}} \approx C_{i j}{ }^{E I 0}+C_{i j}{ }^{E I 1} \alpha+\cdots
$$

With computing each perturbation term of the compliance matrix considering random variation and substituting it into Eq.(2.8), each perturbation term of the equivalent elastic constants can be obtained ${ }^{(13)}$.

Using the first order perturbation-based analysis, the stochastic characteristics such as the expectation and variance of the homogenized equivalent elastic constants can be estimated using the following first order second moment method (FOSM) ${ }^{(16)}$.

$$
\left.\begin{array}{l}
\mathrm{E}\left[E^{E I}\right]=E^{E I 0} \\
\operatorname{Var}\left[E^{E I}\right]=\sum_{i} \sum_{j} E^{E I 1}{ }_{i} E_{j}^{E I 1} \operatorname{cov}\left[\alpha_{i}, \alpha_{j}\right]
\end{array}\right\}
$$

where $\operatorname{cov}\left[\alpha_{i}, \alpha_{j}\right]$ is the covariance with respect to $\alpha$.

\section{Problem Settings}

In this paper, influence of the expected value and random variation of volume fraction of fiber on the stochastic characteristics of a homogenized elastic property of a 
unidirectional composite material is investigated. Four variables related to elastic properties as Young's modulus of fiber $\left(E_{f}\right)$ and matrix $\left(E_{m}\right)$, Poisson's ratio of fiber $\left(v_{f}\right)$ and matrix $\left(v_{m}\right)$, and volume fraction of fiber $\left(V_{f}\right)$ are taken into account as random variables. The expected values of elastic properties for fiber and matrix are listed in Table 1. The properties of fiber and matrix are employed correspond to E-glass and Epoxy resin.

It is assumed that an observed value of a material property or geometrical parameter can be expressed by the following equation.

$$
X^{*}=X^{0}(1+\alpha)
$$

where the superscript * indicates an observed value and the superscript 0 indicates an expected value. Also, it is assumed that the random variables are independently distributed according to the normal distribution. Stochastic characteristics of a stochastic variable $\alpha$ are assumed as

$$
\mathrm{E}[\alpha]=0, \operatorname{Var}[\alpha]=\sigma^{2}
$$

where $\mathrm{E}[\alpha]$ is an expected value and $\operatorname{Var}[\alpha]$ is variance of a stochastic variable $\alpha$. In this case, it is assumed that $\sigma=0.05$.

Table 1 Expected values of elastic properties for fiber and matrix

\begin{tabular}{|c|c|c|}
\hline & Fiber ( E-glass ) & Matrix ( Epoxy ) \\
\hline Young's modulus ( GPa ) & 73.0 & 4.5 \\
\hline Poisson's ratio & 0.22 & 0.39 \\
\hline
\end{tabular}

\section{Numerical Results}

\subsection{Accuracy of the perturbation-based analysis for each volume fraction of fiber}

In this study, the perturbation-based analysis using the equivalent inclusion method (PEI) is used for the stochastic homogenization analysis. Accuracy of the PEI for a certain volume fraction and relationship between the accuracy and a degree of random variation in a material or geometrical property of component materials have been reported by Sakata et al. ${ }^{(13)}$, however, influence of the volume fraction of fiber on the accuracy has not been reported yet. Since this paper discusses a stochastic property for every volume fraction which can be realized using PEI, it should be evaluated at first.

Figures 2-4 show the estimated coefficient of variance (CV) of the equivalent Young's modulus and Poisson's ratio of the composite material considering $E_{f}, v_{m}$ and $V_{f}$ variation. The estimated values using the Monte-Carlo simulation (MC) are obtained as an average of 20th trials of $\mathrm{MC}$, and the number of samples for each trial is 10,000 . The error-bars in the figures indicate the maximum and minimum values obtained from the 20th trials. FOSM in the figures indicates the result obtained from the perturbation-based analysis.

From Figs.2-4, it can be recognized that the most of CVs are well estimated with using the PEI because the estimated results with PEI are located within the error range of each estimation with using MC. Only the estimated result of PEI for $V_{f}$ variation at $V_{f}=0.6$ includes a relatively large estimation error. This is caused by estimation error in the first-order perturbation approximation, but the error is less than $10 \%$, and it can be concluded that PEI will be reliable for stochastic homogenization analysis at each volume fraction of fiber. 


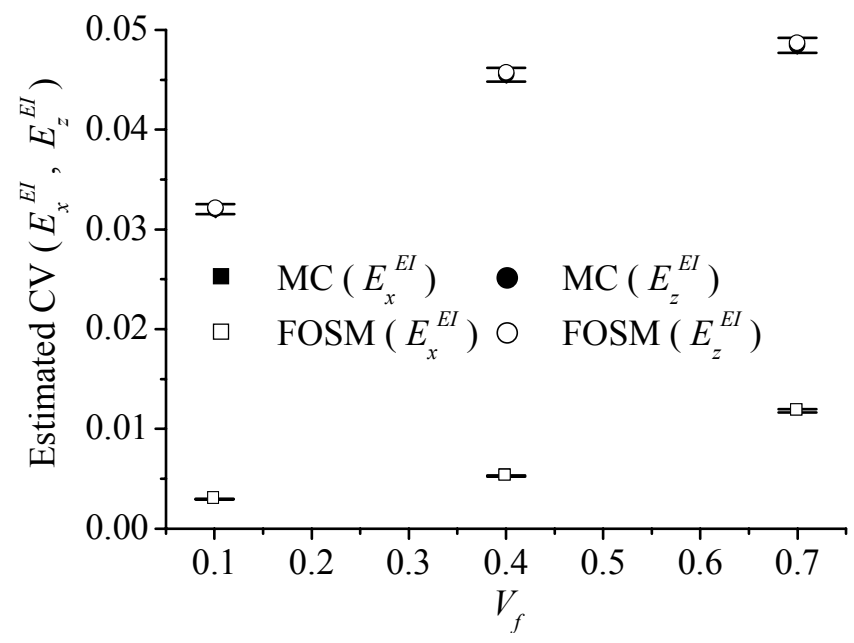

(a) $\mathrm{CV}$ of the equivalent Young's modulus

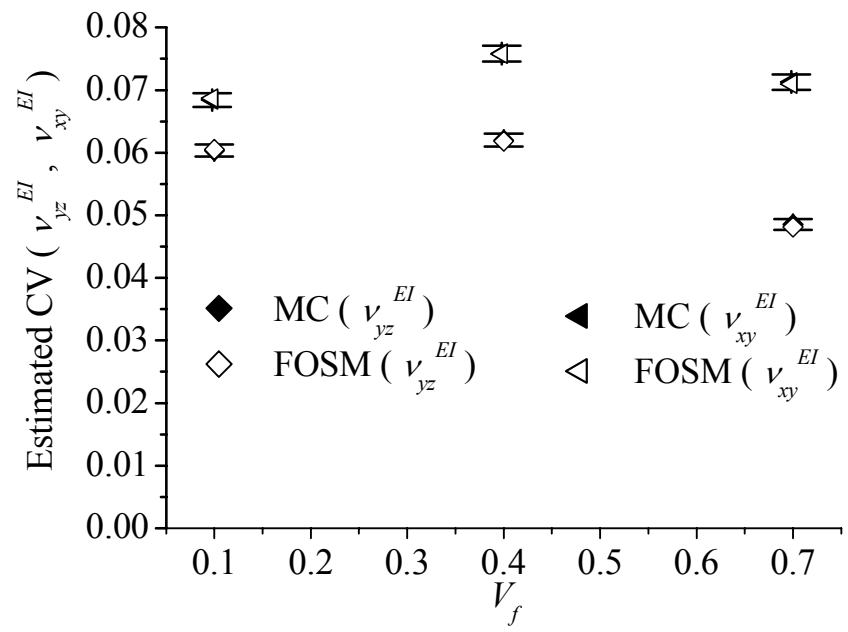

(b) $\mathrm{CV}$ of the equivalent Poisson's ratio

Fig.2 Estimated CV of homogenized elastic properties for $E_{f}$ variation

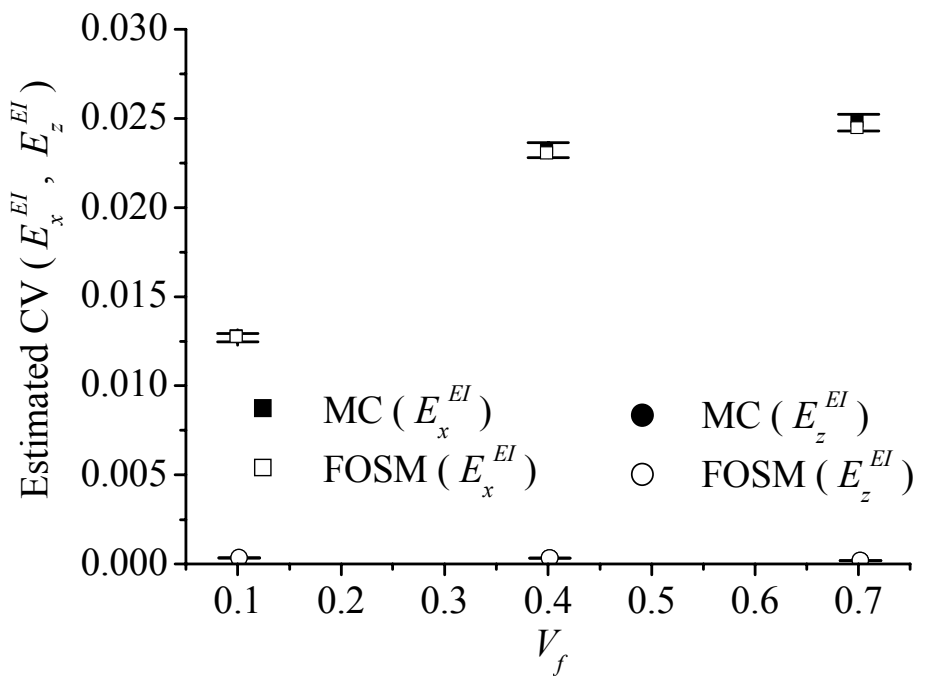

(a) $\mathrm{CV}$ of the equivalent Young's modulus 


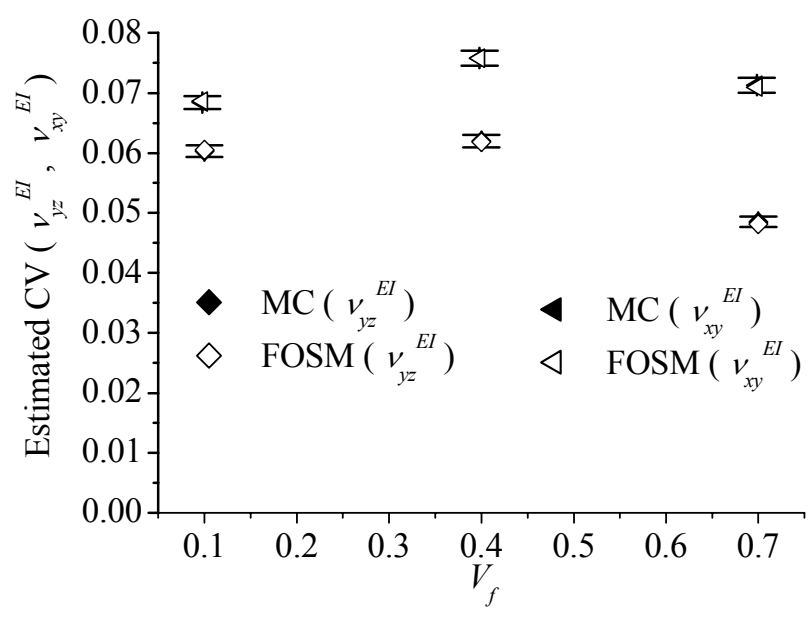

(b) $\mathrm{CV}$ of the equivalent Poisson's ratio

Fig.3 Estimated CV of homogenized elastic properties for $v_{m}$ variation

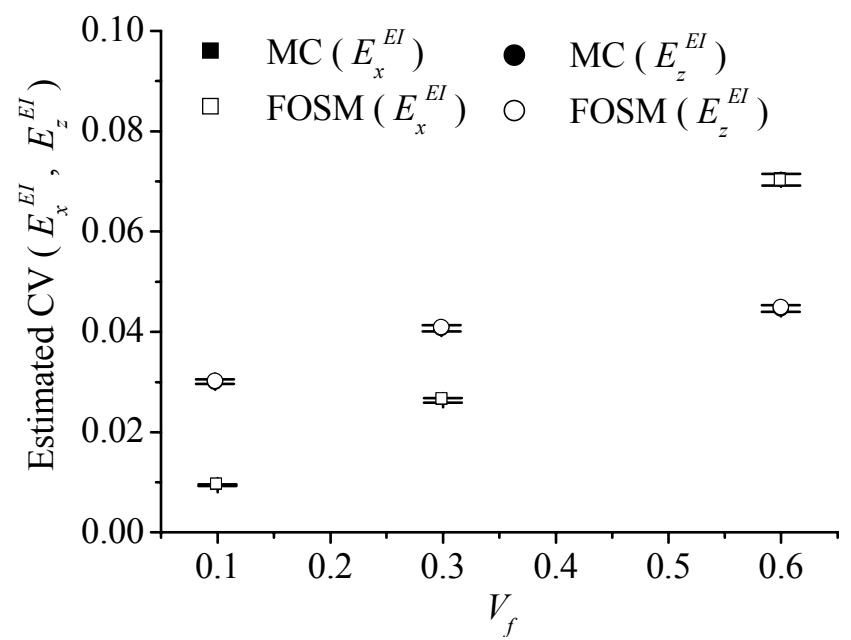

(a) CV of the equivalent Young's modulus

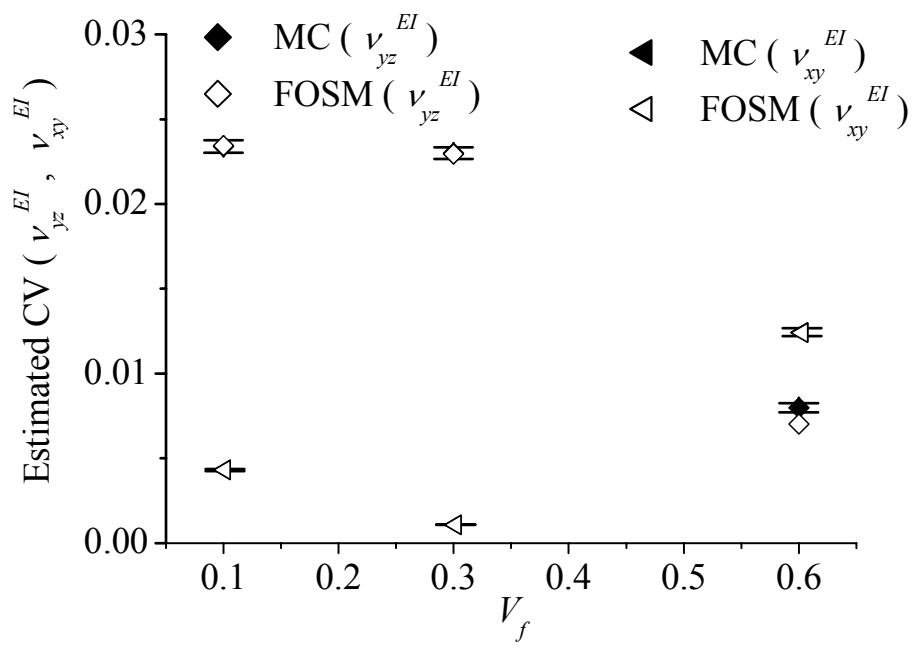

(b) $\mathrm{CV}$ of the equivalent Poisson's ratio

Fig.4 Estimated CV of homogenized elastic properties for $V_{f}$ variation 
4.2 Influence of the $V_{f}$ variation on the stochastic characteristics of the equivalent elastic constants

In this section, influence of the volume fraction and the random variation in volume fraction of fiber on the stochastic characteristic of homogenized elastic properties is investigated. Figures 5 and 6 show the relationships between the volume fraction of fiber and the estimated CVs in case of $E_{f}$ or $v_{m}$ variation. From these figures, it is recognized that $\mathrm{CV}$ of the equivalent Young's modulus and shearing modulus increase as the volume fraction of fiber increases in case of $E_{f}$ variation, while those CVs have a peak for $v_{m}$ variation. CVs of the equivalent Poisson's ratio for both $E_{f}$ and $v_{m}$ variations also have a peak.

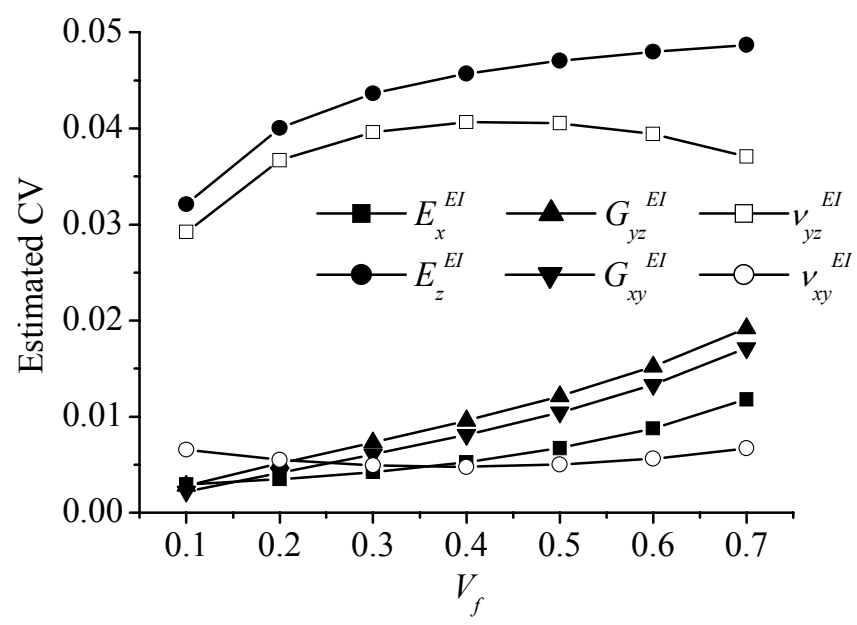

Fig.5 Estimated CV of homogenized elastic properties considering $E_{f}$ variation for each volume fraction of fiber

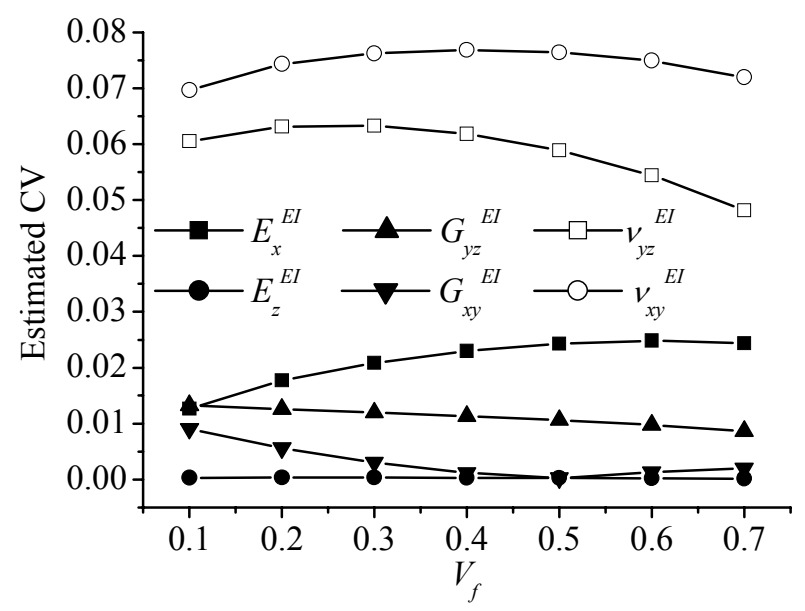

Fig.6 Estimated CV of homogenized elastic properties considering $v_{m}$ variation for each volume fraction of fiber

The CV of the equivalent elastic constants caused by the $V_{f}$ variation for each volume fraction of fiber is also computed using PEI. Figure 7 shows the relationship, and it is recognized that the $\mathrm{CV}$ of the equivalent Young's modulus and shearing modulus increase as the volume fraction increases. This tendency of the relationships between CVs of each equivalent elastic constant and volume fraction of fiber is similar to the CVs for $E_{f}$ variation, but those relationships between the absolute values are different from each other, 
and some of them are larger than that of $E_{f}$ variation. This fact shows necessity of the stochastic homogenization analysis for $V_{f}$ variation in addition to a random variation in elastic properties of component materials.

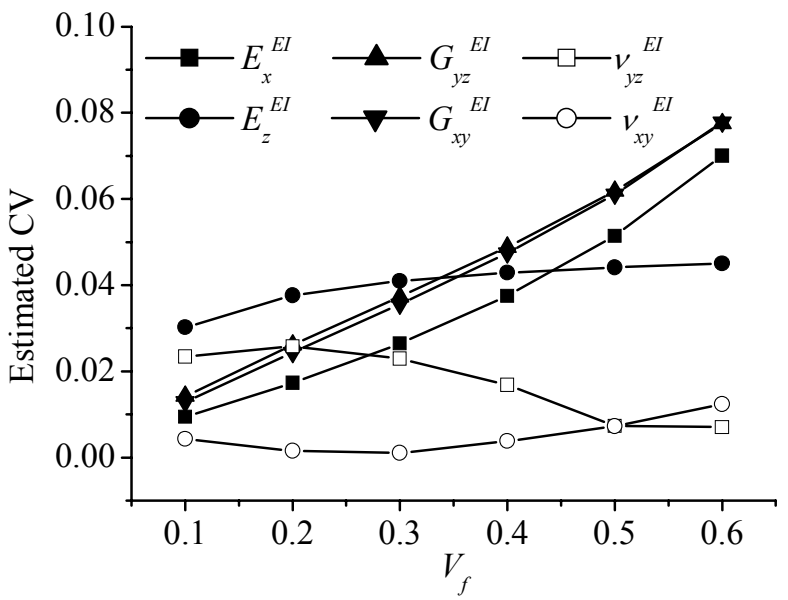

Fig.7 Estimated CV of homogenized elastic properties considering $V_{f}$ variation for each volume fraction of fiber

Additionally, the CVs of the equivalent elastic constants for simultaneous random variations in the material properties of component materials and the volume fraction of fiber are computed using the PEI. Figure 8 shows the result of $\mathrm{CV}$ estimation for the simultaneous random variations. Figure 8 (a) shows the results considering random variations in all elastic properties, namely $E_{f}, E_{m}, v_{f}$ and $v_{m}$ variations, and Fig.8(b) is the result for $V_{f}$ variation in addition to those four elastic property variations.

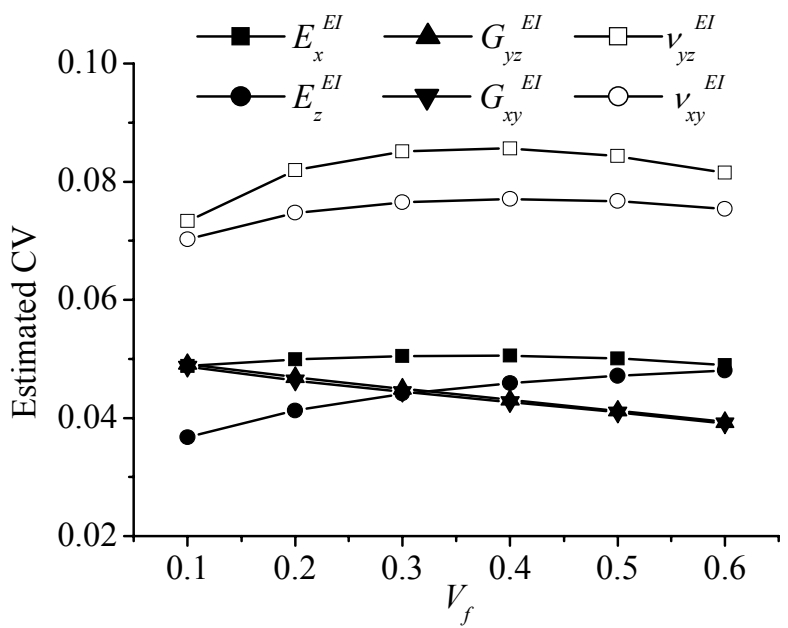

(a) Estimated CV of homogenized elastic constants considering all material properties random variation 


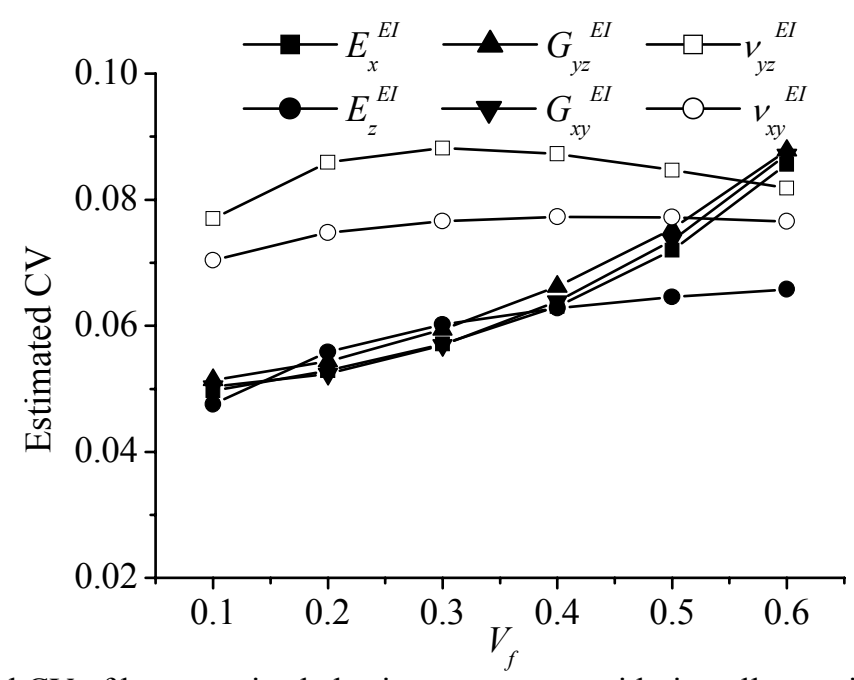

(b) Estimated CV of homogenized elastic constants considering all material properties and geometrical random variation

Fig.8 Estimated CV of homogenized elastic properties considering all material properties and geometry variation for each volume fraction of fiber

From these figures, it is recognized that the relationships between the CVs and the volume fraction of fiber are different from each other, especially for the $\mathrm{CV}$ of the equivalent Young's or shearing modulus. If only the random variations in elastic properties are taken into account, the maximum $\mathrm{CV}$ is about 0.05 , and $\mathrm{CV}$ of the equivalent shearing modulus decreases as the volume fraction increases. On the other hand, the CVs for the equivalent Young's and shearing modulus increase as the volume fraction increase in case that $V_{f}$ variation is taken into account, and the CVs reach about 0.07 or 0.08 when the volume fraction is larger than 0.5 . This result shows that the $V_{f}$ variation has large influence of the $\mathrm{CV}$ of the equivalent Young's modulus and shearing modulus, especially in case of higher volume fraction of fiber, and it should be taken into account when the random variation of the equivalent elastic modulus is evaluated, especially in case of such a unidirectional fiber reinforced composite material analyzed in this example.

\subsection{Effect of controlling a random variation in the volume fraction of fiber}

If we can control or reduce a degree of random variation in the volume fraction of fiber, $\mathrm{CV}$ of the equivalent elastic property such as the equivalent Young's modulus may be also reduced. From this point of view, influence of a degree of the random variation in the volume fraction of fiber on $\mathrm{CV}$ of the equivalent Young's modulus in case of a unidirectional fiber reinforced composite material is discussed with a comparison of CVs of the equivalent elastic constants for each degree of the $V_{f}$ variation.

Figure 9 shows relationships between $\mathrm{CV}$ of the equivalent Young's modulus at each volume fraction of fiber for all random variations described above and a degree of $V_{f}$ variation. Figure 9(a) shows the relationship between $\mathrm{CV}$ of the equivalent $E_{z}$ and the degrees of $V_{f}$ variation, Fig.9(b) shows that for the equivalent $E_{x} . \alpha\left[V_{f}\right]$ in the figures indicates the standard deviation of $V_{f}$. From these figures, it is recognized that the influences of the degree of $V_{f}$ variation on the CVs are different from each other, and CV can be reduced if the degree of $V_{f}$ variation can be appropriately controlled. For instance, if a designer wants to control the CVs to be less than that of the elastic properties of component materials, namely the CVs are less than 0.05 for every volume fraction of fibers, it is recognized that the $\mathrm{CV}$ of $V_{f}$ should be less than about 0.02 in case of controlling $\mathrm{CV}$ of $E_{z}$ or $E_{x}$ as shown in this case. It will be difficult how to reduce or control CV of $V_{f}$ in practice, but this result will indicate one of ways to control $\mathrm{CV}$ of the equivalent elastic constant. 


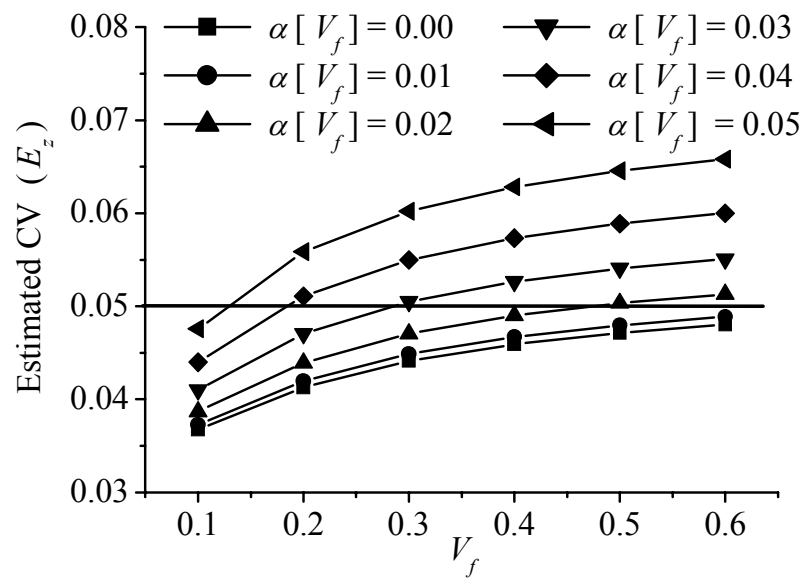

(a) Estimated $\mathrm{CV}$ of the equivalent elastic constant for fiber direction

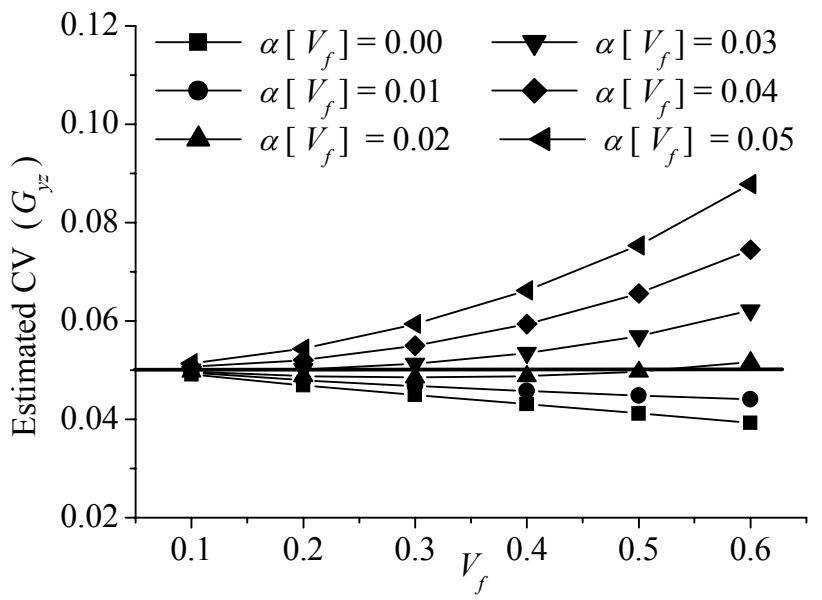

(b) Estimated $\mathrm{CV}$ of the equivalent elastic constant for transverse direction

Fig.9 Estimated CV of homogenized elastic constants considering all material properties and $V_{f}$ variation for each degree of $V_{f}$ variation

\section{Conclusion}

In this paper, influence of volume fraction of fiber and its random variation on the stochastic characteristic of the equivalent elastic constants for random variations in material and geometrical properties of component materials is discussed. The first order perturbation-based stochastic homogenization analysis method using the equivalent inclusion method is applied to the analysis.

At first, accuracy of the perturbation-based method is investigated for various volume fractions of fiber. It can be confirmed from the numerical results that the perturbation-based method will be usable for the analysis.

Next, influence of the volume fraction of fiber and its random variation on the stochastic characteristics of the equivalent elastic constants of a unidirectional fiber reinforced composite material for microscopic material and geometrical uncertainties is investigated. In this example, it is recognized that the volume fraction and its random variation have a large influence on the stochastic homogenization property.

Finally, in order to reduce the random variation in the equivalent Young's modulus, influence of a degree of $V_{f}$ variation on CVs of the equivalent Young's modulus is evaluated. Also, a limit of $V_{f}$ variation in order to control the random variation in the equivalent Young's modulus in case of a unidirectional fiber reinforced composite material is estimated from the CVs for several degrees of $V_{f}$ variation. 


\section{References}

[1] Kaminski, M., Computational Mechanics of Composite Materials: Sensitivity, Randomness and Multiscale Behavior, Springer, (2004).

[2] Ostoja-Starzewski, M., Microstructural Randomness and Scaling in Mechanics of Materials, CRC Press, (2008).

[3] Xu, X . F. and Brady, L. G., Computational stochastic homogenization of random media elliptic problems using Fourier Galerkin method, Finite Elements in Analysis and Design Vol.42 (2005), pp.613-622.

[4] Kaminski, M. and Kleiber, M., Stochastic Structural Interface Defects in Fiber Composites, International Journal of Solids and Structures, Vol.33, No.20-22 (1996), pp.3035-3056.

[5] Sakata, S., Ashida, F., Kojima, T. and Zako M., Influence of Uncertainty in Microscopic Material Property on Homogenized Elastic Property of Unidirectional Fiber Reinforced Composites, Theoretical and Applied Mechanics, Vol.56 (2007), pp.67-76.

[6] Kaminski, M.and Kleiber, M., Perturbation based stochastic finite element method for homogenization of two-phase elastic composites, Computers and Structures, Vol.78 (2000), pp.811-826.

[7] Kaminski, M., Stochastic finite element method homogenization of heat conduction problem in fiber composites, Structural Engineering and Mechanics, Vol.11, No.4 (2001), pp. 373-392.

[8] Sakata, S., Ashida, F., Kojima, T. and Zako, M., Three-dimensional stochastic analysis using a perturbation-based homogenization method for elastic properties of composite material considering microscopic uncertainty, International Journal of Solids and Structures, Vol.45, No.3-4 (2008), pp.894-907.

[9] Sakata, S., Ashida, F. and Zako, M., Stochastic Response analysis of FRP using the Second-order Perturbation-based Homogenization Method, Journal of Solid Mechanics and Materials Engineering, Vol.2, No.1 (2008), pp.70-81,.

[10] Sakata, S., Ashida, F. and Zako, M., Kriging-based Approximate Stochastic Homogenization Analysis for Composite Material, Computer Methods in Applied Mechanics and Engineering, Vol.197, issues21-24 (2008), pp.1953-1964.

[11] Kaminski, M., Sensitivity and randomness in homogenization of periodic fiber-reinforced composites via the response function method, International Journal of Solids and Structures, Vol. 46, Issues 3-4, (2009), pp.923-937.

[12] Sakata, S., Ashida, F. and Kojima, T., Perturbation Analysis for Stochastic Homogenization Problem of Fiber Reinforced Composites with the Equivalent Inclusion Method, International Journal of Solid and Structures, Vol.45, No.25-26 (2008), pp.6553-6565.

[13] Sakata, S., Ashida, F. and Kojima, T., On perturbation-based stochastic homogenization analysis of homogenized equivalent elastic constants using equivalent inclusion method, Journal of Solid Mechanics and Materials Engineering, Vol.3,No.3 (2009), pp.541-551.

[14] Mori, T. and Tanaka, K., Average Stress in Matrix and Average Elastic Energy of Materials With Misfitting Inclusions, Acta Metallurgica, Vol.21 (1972), pp.571-574.

[15] Zako, M. and Matsumoto, K., Behavior Analysis of Composite Materials, Asakura-shoten, (1998), pp.19-20. (in Japanese)

[16] Nakagiri, S. and Hisada, T., Introduction to the Stochastic Finite Element Method, Baifu-kan, (1985), p.23. (in Japanese) 